\title{
ADAMTS4 or ADAMTS5: Which is the Key Enzyme in the Cartilage Degradation of Osteoarthritis and Kashin-Beck Disease?
}

\section{Peilin Meng}

Xi'an Jiaotong University, National Health and Family Planning Commission

Mikko J. Lammi

Umeå University

\section{Linlin Yuan}

Xi'an Jiaotong University, National Health and Family Planning Commission

\section{SiJia Tan}

Xi'an Jiaotong University, National Health and Family Planning Commission

\section{Feng'e Zhang}

Xi'an Jiaotong University, National Health and Family Planning Commission

\section{Peilin Li}

Xi'an Jiaotong University, National Health and Family Planning Commission

\section{Yanan Zhang}

Xi'an Jiaotong University, National Health and Family Planning Commission

\section{Wenyu Li}

Xi'an Jiaotong University, National Health and Family Planning Commission

\section{Sen Wang ( $\nabla$ senwang2016@163.com )}

Xi'an Jiaotong University, National Health and Family Planning Commission

\section{Xiong Guo}

Xi'an Jiaotong University, National Health and Family Planning Commission

\section{Research Article}

Keywords: ADAMTS4, ADANTS5, Osteoarthritis, Kashin-Beck Disease, immunohistochemical.

Posted Date: June 30th, 2021

DOI: https://doi.org/10.21203/rs.3.rs-640139/v1

License: (c) (1) This work is licensed under a Creative Commons Attribution 4.0 International License. Read Full License 


\section{Abstract}

Background: This study aims to investigate the altered expression of a disintegrin and metalloproteinase with thrombospondin motifs 4囚ADAMTS4『and a disintegrin and metalloproteinase with thrombospondin motifs 5囚ADAMTS5『in the human articular cartilage between osteoarthritis(OA) and Kashin-Beck disease $(K B D)$ and compare their roles in the cartilage injury.

Methods: Articular samples were collected from confirmed OA patients and KBD patients then divided into three groups, and the articular cartilages from the normal donors were used as controls. The morphology 8 location and expression of ADAMTS4 and ADAMTS5 as well as aggrecan were detected by histochemical staining and immunohistochemical staining.

Results: Compared to the control, the number of living cells in OA and KBD groups declined at three zones. Meanwhile, the results of toluidine blue staining showed that there was a loss of aggrecan in extracellular matrix of KBD and OA cartilages. The amounts of chondrocytes positively stained for ADAMTS4 were lower in the middle and deep zones of OA and KBD cartilages than those in the control samples. On the contrary, the immunostaining for ADAMTS5 significantly increased in OA and KBD group compared with control group at all three zones. Notably, although there was no statistical significance, the expression of ADAMST5 in KBD was slightly higher than the ones in OA in superficial, middle and deep zones.

Conclusions: The role ADAMTS4 acted in aggrecan degradation was seemingly minor. It could be inferred that ADAMTS5 is the key enzyme in the cartilage destruction, particularly in KBD cartilage, which could explain the complex pathogenesis of KBD and provide a potential therapeutic target for KBD patients.

\section{Background}

Osteoarthritis $(\mathrm{OA})$ is the most prevalent degenerative joint disease in the world. As the main cause of chronic pain and disability in the senior citizens, it is clinically characterized by stiffness, arthralgia, and limitation of movement(1). Kashin-Beck disease (KBD) is an endemic, chronic osteochondropathy, which can directly result in the joint deformity, dwarfism, depression and even disability(2), posing an increasing burden and challenge on society and individual. In China, this disease is widespread from northeast to the southwest, it can also be found in some regions of Eastern Siberia in Russia and in North Korea. Three hypotheses have been formulated to explain the occurrence of KBD: 1) an endemic deficiency of Selenium, 2) a cereal contamination by mycotoxins and 3) high humic acid levels in drinking water (3-5). Despite extensive investigations, none of these has been verified by epidemiological and experimental evidences. Thus, KBD is still regarded to have a multi-factorial etiology and complex pathogenesis.

Through reviewing previous studies, a conclusion can be drawed that KBD shares its clinical symptoms and pathology with $\mathrm{OA}$, particularly at earlier stages of the disease, although gene expression and pathogenesis of KBD have been indicated to differ from $\mathrm{OA}(6,7)$. For example, painful joints, narrowed joint space, osteophytes, and movement restrictions of joints can be observed in the cartilage from both 
$\mathrm{OA}$ and KBD patients. Some pathological changes, such as predominant degradation of extracellular matrix (ECM) in affected articular cartilage, apoptosis and necrosis of chondrocytes overlap in OA and KBD. Given this, KBD should be undoubtedly viewed as a specific type of OA.

The imbalance of the synthesis and degradation of ECM is one of the important causes in the cartilage destruction. The chondrocytes maintain the cartilage structure and the homeostasis of the cellular environment by synthetizing and catabolizing ECM macromolecules. The amount of cartilage matrix will decline, if the efficiency of degradation of ECM surpass their synthesis, and eventually leads to the destruction of cartilage(8). Aggrecan is the important component of ECM, imparting the compressive ability for cartilage(9). The depletion of aggrecan is a hallmark event happening in the advanced stage of $\mathrm{OA}$, which is mediated by aggrecanases. Aggrecanases are the principal proteinases, present in the articular cartilage and growth plate cartilage, which belong to the a disintegrin and metalloproteinase with thrombospondin motifs (ADAMTS) family. As the indispensable members of ADAMTS, recent studies have indicated that ADAMTS4 and ADAMTS5 are responsible for the degradation of aggrecan in human OA models(10-12). It is plausible that ADAMTS4 and ADAMTS5 make major contributions to the loss of proteoglycans during the progression of KBD.

In this study, the presences of ADAMTS4, ADAMTS5 and aggrecan were tested by immunohistochemical stainings of cartilage from the patients of KBD and $\mathrm{OA}$, as well as the normal group. The comparison between OA, KBD and the normal groups could not only clarify which one of ADAMTS4 or ADAMTS5 is the key aggrecanase in cartilage depletion, but also explore the roles of these aggrecanases in the pathogenesis of KBD, which will be helpful to illustrate the etiology of KBD and put forward new potential therapeutic targets for KBD.

\section{Materials And Methods}

\section{Samples and materials}

OA patients ( $n=9$, age: $45-65$ years old, sex ratio: $M / F=4 / 5)$ and KBD patient( $n=11$, age: $50-63 y e a r s$, sex ratio: $M / F=8 / 3$ ) were primarily enrolled from those who underwent surgical treatment. All KBD patients were diagnosed and confirmed according to Chinese diagnosis criterion(13). The normal cartilage samples were collected from the patients who suffered from traffic accidents or polydactyly treatment surgically ( $n=12$, age: $37-70 y e a r s$, sex ratio: $M / F=5 / 7)$. All normal samples are from donors living in nonKBD endemic areas. The detailed information about subjects is shown in the Table 1.

This study was permitted by medical research permission granted by the Human Ethical Committee in Xi'an Jiaotong University. All the participants were informed of this studies, after which they provided a written informed consent.

\section{Antibodies}


The primary antibodies applied were as follows: ADAMTS4(catalogue no. ab185722, Abcam, Cambridge, England), ADAMTS5(catalogue no. ab182795, Abcam, Cambridge, England). The specificities of immunohistochemical method of these antibodies have been widely validated(14-16).

\section{Immunohistochemical staining}

These cartilage tissues fixed in the $4 \%$ paraformaldehyde were decalcified, embedded in paraffin within $8 \mathrm{~h}$ after surgery, then cut in coronary direction into $5 \mu \mathrm{m}$ thickness after dehydration, and prepared for the applications described below. For deparaffinization, $5 \mu \mathrm{m}$ sections were immersed in xylene and rehydrated with a series of gradually decreasing concentrations of ethanol( $100 \%-80 \%)$, then incubated with $3 \%$ hydrogen peroxide $\mathrm{H}_{2} \mathrm{O}_{2}$ for $15 \mathrm{~min}$ at room temperature. After rinsing with phosphate-buffered saline (PBS) for three times, the sections were incubated $2 \mathrm{mg} / \mathrm{ml}$ hyaluronidase for $20 \mathrm{~min}$. After incubation in blocking buffer (the normal goat serum, from Zhong Shan Gold Bridge Rabbit SP reagent, Beijing Zhong Gold Bridge Biological Technology Co., 18112A11) for 20 min, the samples were incubated with the primary antibodies of ADAMTS4 at 1:200 dilutions and ADAMTS5 at 1:50 dilutions in 1\% bovine serum albumin in PBS at $4{ }^{\circ} \mathrm{C}$ overnight. The primary antibody of negative control was replaced by PBS. Then the sections were incubated with horseradish peroxidase-conjugated goat anti-rabbit lg $\mathrm{G}$ at $37^{\circ} \mathrm{C}$ for 20 min. After rinsing in PBS, hematoxylin counterstaining was performed. For visualization, a diaminobenzidine (DAB) kit (ZLI-9017, ZSGB-BIO, China) was applied. Finally, dehydration, clearing and sealing coverslips were performed.

\section{Hematoxylin and eosin (H\&E) and toluidine blue (TB) staining}

For hematoxylin-eosin (H\&E) and toluidine blue (TB) stainings the deparaffinization followed the procedure used for immunohistochemical samples. Then sections were stained with H\&E or $0.1 \%(\mathrm{w} / \mathrm{v})$ TB dye solution. After washing with running water, dehydrated by a series of gradually increasing concentrations of ethanol solution ( $80-100 \%)$ and xylene, then samples were sealed by coverslips.

\section{Image capture and quantification}

The stained section images were acquired by a Leica SCN400 slide scanner (Leica Microsystems GmbH, Wetzlar, Germany) under a 100x magnification field, then three representative regions were randomly selected from superficial, middle and deep zones were quantified by Image $J$ software (NIH, USA). The positive staining rate was calculated as follows:

The positive staining cell rate $=$

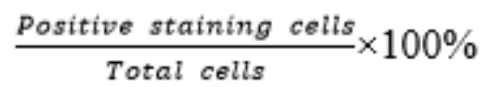

\section{Statistical analysis}

SPSS 22.0 software (SPSS Inc, USA) was applied for the data entry and statistical analysis. If the data from $\mathrm{OA}, \mathrm{KBD}$ and control groups were in accordance with normal distribution by the test for normality and equal variance, the rank-based ANOVA test was used to examine the statistical discrepancy of 
positive staining cell rate among $\mathrm{OA}, \mathrm{KBD}$ and control groups. If not, nonparametric rank sum test was used, and all statistical tests were bilateral tests. $P \otimes 0.05$ was regarded for statistically significant difference.

\section{Results}

\section{H\&E staining}

H\&E staining manifested that the quantity of living cells of OA (superficial zone, $P \otimes 0.001$, middle zone, $P=0.001$, deep zones, $P=0.003$ ) and KBD (superficial zone, $P \otimes 0.001$, middle zone, $P=0.004$, deep zones, $P \square$ $0.001)$ declined evidently among three zones compared to the control group. This suggested that the cartilage may undergo a focal chondrocyte death (necrosis), which is associated with proteoglycans depletion. In the superficial zones, the number of living cells of OA were less than $\operatorname{KBD}(P=0.036)$ (Fig.1.).

\section{TB staining}

TB staining was applied to examine the concent of ECM in cartilage. The results of TB staining showed a significant decrease in superficial $(P=0.021)$, middle $(P=0.021)$, deep zones $(P=0.027)$ of $\operatorname{KBD}$ cartilage in comparison to healthy cartilage, whereas the percentage of intensive staining of $O A$ cartilage was distinctly lower compared to the control only in superficial zone( $P=0.034)$ (Fig2.).

\section{IHC staining}

Theoretically, the expression aggrecanases should increase in OA and KBD cartilage. However, there were no difference ADAMTS4 stainings in the superficial zone among the three groups. In the middle zone, the positive staining rate of ADAMTS4 in OA cartilage reduced compared to control one $(P=0.001)$. Similarly, this kind of decrease also happened in the deep zone in both OA and KBD cartilage $(P=0.008, P<0.001)$. As for the comparison of expression of ADAMTS4 between KBD and OA cartilage, the positive staining rates in KBD group had a rise when compared to OA in both superficial and middle zones, wherein the statistically difference were observed in the middle zones $(P=0.009)$. In the deep zone, condition changed: as the number of positive staining cells in KBD were lower than in OA(Fig3.).

The immunostaining of ADAMTS5 were much stronger in OA and KBD group than those in control group in the superficial zone ( $P \otimes 0.001, P \otimes 0.001)$. Meanwhile, the positive staining cells in the middle zones of $\mathrm{OA}$ and KBD also had an obviously increase compared to the healthy cartilage $(P=0.003, P \otimes 0.001)$. In the deep zone, the distribution of positive staining cells in OA and KBD were more intensive than in the control ( $P \otimes 0.001, P \otimes 0.001)$. Totally, there was a tendency that the expression of ADAMTS5 in KBD was slightly higher than $\mathrm{OA}$ among all three zones in cartilage, although without statistical difference (Fig4.).

\section{Discussion}


Apoptosis, necrosis, chondrocytes aging and loss of proteoglycans were universally acknowledged as the primary features of cartilage injure(17-21), As direct consequences of these, the density of the living cells in the cartilage decreases. The results of H\&E staining verified that such a phenomenon really was obvious in the cartilage of $\mathrm{OA}$ and $\mathrm{KBD}$.

Proteoglycans are complex macromolecules distributing in the ECM of articular cartilage, having a protective effect on joints. Dominant proteoglycans in cartilage are aggrecan (22). Increasing number of research has demonstrated that there is a close association between loss of aggrecan and cartilage diseases(23-25).It could be observed in the TB staining that the staining intensity of proteoglycans in KBD was quite low compared to OA group and control group, which showed that the depletion of aggrecan is more serious in cartilage of KBD.

It is generally accepted that two families of metalloproteases are the major contributors in the progression of degradation of ECM: matrix metalloproteinase (MMP) family and ADAMTS family(26). However, in comparison to the MMPs, aggrecanases seemingly have more association with the depletion of aggrecan, because it was the greatest in areas of cartilage adjacent to sites of cartilage erosion(25).

When referring to the roles of ADAMTS family played in the degradation of aggrecan, researchers often pay more attention to the ADAMTS4 and ADAMTS5, since other members of family related to degradation of aggrecan, such as ADAMTS1, ADAMTS8, ADAMTS9, has been shown to have a rather low aggrecan-degrading activity $(27,28)$. ADAMTS4 and ADAMTS5 leave the aggrecan core protein at the aggrecanase-specific Glu373- Ala374 bond in the interglobular domain (IGD) region(29, 30), therefore, they have been regarded as the key aggrecanase. ADAMTS4 and ADAMTS5 have similar structure, containing a disintegrin domain, a thrombospondin domain, a cysteine-rich domain, and a spacer domain, but ADAMTS5 has an additional TS domain after the spacer domain. A study reported that in the IGD region the aggrecanolytic activity of ADAMTS5 are fourfold compared to ADAMTS4, in the CS-2 region of aggrecan 2.5 fold, and under physiological conditions the aggrecanase activity of ADAMTS5 was at least 1000 -fold greater than that of ADAMTS4 $(31,32)$.

Based on the research of activity and molecular basis of ADAMTS4 and ADAMTS5, advanced works from lots of laboratories have further illustrated the impact of aggrecanase on increase of aggrecan loss related to cartilage diseases in different ways(33-36). Although there are some reviews comprehensively summarizing the regulation of ADAMTS4 and ADAMTS5 in OA(26, 37-39), it is still controversial that which of them is the main aggrecanaes in the destruction of cartilage. Some reports argue that ADAMTS4 is primarily expressed in an active form in osteoarthritic cartilage, while ADAMTS 5 would be constitutively expressed in both normal and OA cartilage(40).

Others suggested that owing to the high affinity of its non-catalytic domains for glycosaminoglycan chains, ADAMTS5 is the most active aggrecanase to increase the loss of aggrecan(32). It has also been proposed that ADAMTS4 is the most inducible aggrecanase upon cytokine stimulation, whereas ADAMTS5 is the most abundant aggrecanase(41), which manifests that ADAMTS5 is constitutive. In the murine models of OA, ADAMTS5 deficiency could protect mice from OA, but deletion of ADAMTS4 didn't 
have any influence on the normal growth and physiology of mice $(42,43)$. As discussed above, ADAMTS5 appears to play the key role in the cartilage depletion, though these conclusions are limited to animal model, and the discrepancy between human and murine should not been ignored. Therefore, aims of this study not only investigated the main aggrecanases involving the cartilage degradation, but also explored whether ADAMST4 and ADAMTS5 play the equivalent roles in the progression of KBD.

In our study, the expression of ADAMTS4 is not in accordance with the points of some previous studies: the percentage of positive staining of the control was more intensive than $\mathrm{OA}$ and KBD in the middle and the deep zones. Generally speaking, ADAMTS4 should be overexpressed in the damaged cartilage. However, the mRNA expression of ADAMTS4 was significantly repressed at the early stage of OA(44). Besides, in the superficial zone, the expressions of ADAMTS4 of OA and KBD had a similar proportion with the control. These results indicated that ADAMTS4-induced cartilage degradation maybe not happened at the early stage of cartilage diseases, and the role of ADAMTS4 is less significant, especially in the superficial zone.

Nevertheless, the expression of ADAMTS5 in OA and KBD were greatly increased compared to the control, particularly in the cartilages from KBD patients. In addition, there was gradual upregulation of ADAMTS5 in the OA group from superficial to deep zone, while the expression in KBD group down-regulated in the deep zone. It confirmed that ADAMTS5 was the crucial aggrecanase, which led to the cartilage damage. This adverse effect is evidence in the deep zone of OA cartilage. What's more, the expression of ADAMTS5 in KBD group was higher than the one in OA group. Based on the results of TB staining of the aggrecan positive cell rates among three groups, it could be inferred that the increased expression of ADAMTS5 is more likely to cause the decrease of aggrecan in OA and KBD.

However, mainly owing to the ethical issue, it was hard to collect enough cartilage from KBD patients. It couldn't manifest adequately the casual relationships between cartilage degradation and ADAMTS5. In order to investigate the role of ADAMTS5 in the cartilage disease, increasing amount of research is required, which could further explain the complex pathogenesis of KBD and provide a potential therapeutic target for KBD patients.

In conclusion, we found that the function of ADAMTS4 in degradation aggrecan is seemingly slight. Hence, we prefer to favor of the opinion that ADAMTS5 is the key enzyme in the cartilage destruction, in particularly in KBD cartilage. Most of previous studies have focused on the roles of ADAMTS4 and ADAMTS5 in OA, while it is rare to investigate the importance of ADAMTS4 and ADAMTS5 in KBD. The test of the expression of ADAMTS4 and ADAMTS5 in OA and KBD cartilage further affirmed our standpoint that ADAMTS5 was more significant in the loss of aggrecan and cartilage damage.

\section{Declarations}

Funding This research was supported by the national natural science fund (No: 81620108026). 
Conflicts of interest/Competing interests The authors declare no conflict of interest. The funders had no role in the design of the study, in the collection, analyses, or interpretation of data, in the writing of the manuscript, or in the decision to publish the results.

Ethics approval and consent to participate The experimental protocol was established, according to the ethical guidelines of the Helsinki Declaration and was approved by the Human Ethics Committee of Xi'an Jiaotong University. Written informed consent was obtained from individual or guardian participants.

Consent for publication Not applicable.

Availability of data and material All data generated or analysed during this study are included in this published article

Code availability Not applicable

Authors' contributions All authors have made substantial contributions to the conception or design of the work, or the acquisition, analysis, or interpretation of data for the work, M.P.L and W.S. have drafted the work or revised it critically for important intellectual content, M.P.L, Z.F.E and L.P.L. have performed the staining, M.P.L., Y.L.L. and T.S.J have organized and analyzed the data, Mikko J. Lammi has edited this manuscript, S.W. and G.X. have approved the final version to be published, S.W. and G.X. agree to be accountable for all aspects of the work in ensuring that questions related to the accuracy or integrity of any part of the work are appropriately investigated and resolved.

\section{References}

1. Pereira D, Ramos E, Branco J. Osteoarthritis. Acta medica portuguesa. 2015,28(1):99-106.

2. Xiong G. Diagnostic, clinical and radiological characteristics of Kashin-Beck disease in Shaanxi Province, PR China. International Orthopaedics. 2001,25(3):147-50.

3. AnPeng, ChunlinYang, HaifenRui, HuLi. Study on the pathogenic factors of kashinâbeck disease. Journal of Toxicology \& Environmental Health. 1992,35(2):79-90.

4. Chasseur, and, Suetens, and, Nolard, and. Fungal contamination in barley and Kashin-Beck disease in Tibet.

5. Peng A, Wang WH, Wang CX, Wang ZJ, Rui HF, Wang WZ, et al. The role of humic substances in drinking water in Kashin-Beck disease in China. Environmental Health Perspectives.107(4):293-6.

6. Duan C, Guo X, Zhang X-D, Yu H-J, Yan H, Gao Y, et al. Comparative analysis of gene expression profiles between primary knee osteoarthritis and an osteoarthritis endemic to Northwestern China, Kashin-Beck disease.62(3):771-80.

7. Zhang F, Guo X, Duan C, Wu S, Yu H, Lammi M. Identification of differentially expressed genes and pathways between primary osteoarthritis and endemic osteoarthritis (Kashin-Beck disease). Scandinavian Journal of Rheumatology. 
8. Wieland HA, Michaelis M, Kirschbaum BJ, Rudolphi KA. Osteoarthritis - An untreatable disease? Nature Reviews Drug Discovery. 2005,4(4):331-44.

9. Bernhard JC. Synthesis of an aggrecan mimic for osteoarthritis specific cartilage treatment: Purdue University, 2011.

10. Verma P, Dalal DK. ADAMTS-4 and ADAMTS-5: Key enzymes in osteoarthritis. Journal of Cellular Biochemistry.112(12):3507-14.

11. Song RH, Tortorella MD, Malfait AM, Alston JT, Yang Z, Arner EC, et al. Aggrecan degradation in human articular cartilage explants is mediated by both ADAMTS-4 and ADAMTS-5. Arthritis \& Rheumatology. 2007,56(2):575-85.

12. Gao SG, Zeng C, Song Y, Tian J, Cheng C, Yang T, et al. Effect of osteopontin on the mRNA expression of ADAMTS4 and ADAMTS5 in chondrocytes from patients with knee osteoarthritis. Experimental \& Therapeutic Medicine.

13. Xiong G. Diagnostic, clinical and radiological characteristics of Kashin-Beck disease in Shaanxi Province, PR China. International orthopaedics. 2001,25(3):147-50.

14. Zhang C, Chiu KY, Chan BPM, Li T, Wen C, Xu A, et al. Knocking out or pharmaceutical inhibition of fatty acid binding protein 4 (FABP4) alleviates osteoarthritis induced by high-fat diet in mice. Osteoarthritis and cartilage. 2018,26(6):824-33.

15. Wang T, Liu Y, Wang Y, Huang X, Zhao W, Zhao Z. Long non-coding RNA XIST promotes extracellular matrix degradation by functioning as a competing endogenous RNA of miR-1277-5p in osteoarthritis. International journal of molecular medicine. 2019,44(2):630-42.

16. Gou Y, Tian F, Dai M, Li H, Lv Q, Kong Q, et al. Salmon calcitonin exerts better preventive effects than celecoxib on lumbar facet joint degeneration and long-term tactile allodynia in rats. Bone. 2019,127:17-25.

17. Zhang $Y$, He Y, Zhang D, Zhang M, Wang M, Zhang Y, et al. Death of chondrocytes in Kashin-Beck disease: Apoptosis, necrosis or necroptosis? International journal of experimental pathology. 2018,99(6):312-22.

18. Hwang HS, Kim HA. Chondrocyte Apoptosis in the Pathogenesis of Osteoarthritis. International journal of molecular sciences. 2015,16(11):26035-54.

19. Chen CT, Burton-Wurster N, Borden C, Hueffer K, Bloom SE, Lust G. Chondrocyte necrosis and apoptosis in impact damaged articular cartilage. Journal of orthopaedic research : official publication of the Orthopaedic Research Society. 2001,19(4):703-11.

20. Loeser RF, Collins JA, Diekman BO. Ageing and the pathogenesis of osteoarthritis. Nature reviews Rheumatology. 2016,12(7):412-20.

21. van Geffen EW, van Caam APM, Schreurs W, van de Loo FA, van Lent $P$, Koenders MI, et al. IL-37 diminishes proteoglycan loss in human OA cartilage: donor-specific link between IL-37 and MMP-3. Osteoarthritis and cartilage. 2019,27(1):148-57.

22. Li S, Cao J, Caterson B, Hughes CE. Proteoglycan metabolism, cell death and Kashin-Beck disease. Glycoconjugate journal. 2012,29(5-6):241-8. 
23. Krishnan Y, Grodzinsky AJ. Cartilage diseases. Matrix biology : journal of the International Society for Matrix Biology. 2018,71-72:51-69.

24. Neame PJ, Sandy JD. Cartilage aggrecan. Biosynthesis, degradation and osteoarthritis. The Journal of the Florida Medical Association. 1994,81(3):191-3.

25. Mort JS, Geng Y, Fisher WD, Roughley PJ. Aggrecan heterogeneity in articular cartilage from patients with osteoarthritis. BMC musculoskeletal disorders. 2016,17:89.

26. Troeberg L, Nagase H. Proteases involved in cartilage matrix degradation in osteoarthritis. Biochimica et biophysica acta. 2012,1824(1):133-45.

27. Zeng W, Corcoran C, Collins-Racie LA, Lavallie ER, Morris EA, Flannery CR. Glycosaminoglycanbinding properties and aggrecanase activities of truncated ADAMTSs: comparative analyses with ADAMTS-5, $-9,-16$ and -18. Biochimica et biophysica acta. 2006,1760(3):517-24.

28. Porter S, Clark IM, Kevorkian L, Edwards DR. The ADAMTS metalloproteinases. The Biochemical journal. 2005,386(Pt 1):15-27.

29. Abbaszade I, Liu RQ, Yang F, Rosenfeld SA, Ross OH, Link JR, et al. Cloning and characterization of ADAMTS11, an aggrecanase from the ADAMTS family. The Journal of biological chemistry. 1999,274(33):23443-50.

30. Tortorella MD, Malfait AM, Deccico C, Arner E. The role of ADAM-TS4 (aggrecanase-1) and ADAMTS5 (aggrecanase-2) in a model of cartilage degradation. Osteoarthritis and cartilage. 2001,9(6):53952.

31. Gendron C, Kashiwagi M, Lim NH, Enghild JJ, Thøgersen IB, Hughes C, et al. Proteolytic activities of human ADAMTS-5: comparative studies with ADAMTS-4. The Journal of biological chemistry. 2007,282(25):18294-306.

32. Fushimi K, Troeberg L, Nakamura H, Lim NH, Nagase H. Functional differences of the catalytic and non-catalytic domains in human ADAMTS-4 and ADAMTS- 5 in aggrecanolytic activity. The Journal of biological chemistry. 2008,283(11):6706-16.

33. Takahata Y, Nakamura E, Hata K, Wakabayashi M, Murakami T, Wakamori K, et al. Sox4 is involved in osteoarthritic cartilage deterioration through induction of ADAMTS4 and ADAMTS5. FASEB journal : official publication of the Federation of American Societies for Experimental Biology. 2019,33(1):61930.

34. Gao SG, Zeng C, Song Y, Tian J, Cheng C, Yang T, et al. Effect of osteopontin on the mRNA expression of ADAMTS4 and ADAMTS5 in chondrocytes from patients with knee osteoarthritis. Experimental and therapeutic medicine. 2015,9(5):1979-83.

35. Busschers E, Holt JP, Richardson DW. Effects of glucocorticoids and interleukin-1 beta on expression and activity of aggrecanases in equine chondrocytes. American journal of veterinary research. 2010,71(2):176-85.

36. Arner EC. Aggrecanase-mediated cartilage degradation. Current opinion in pharmacology. 2002,2(3):322-9. 
37. Bondeson J, Wainwright $\mathrm{S}$, Hughes $\mathrm{C}$, Caterson $\mathrm{B}$. The regulation of the ADAMTS4 and ADAMTS5 aggrecanases in osteoarthritis: a review. Clinical and experimental rheumatology. 2008,26(1):139-45.

38. Verma P, Dalal K. ADAMTS-4 and ADAMTS-5: key enzymes in osteoarthritis. Journal of cellular biochemistry. 2011,112(12):3507-14.

39. Li C, Cao YP, Guan ZP, Huang de Y, Ge ZG. [Aggrecanases and osteoarthritis]. Beijing da xue xue bao Yi xue ban = Journal of Peking University Health sciences. 2009,41(5):611-2.

40. Naito S, Shiomi T, Okada A, Kimura T, Chijiiwa M, Fujita Y, et al. Expression of ADAMTS4 (aggrecanase-1) in human osteoarthritic cartilage. Pathology international. 2007,57(11):703-11.

41. Durigova M, Troeberg L, Nagase H, Roughley PJ, Mort JS. Involvement of ADAMTS5 and hyaluronidase in aggrecan degradation and release from OSM-stimulated cartilage. European cells \& materials. 2011,21:31-45.

42. Glasson SS, Askew R, Sheppard B, Carito BA, Blanchet T, Ma HL, et al. Characterization of and osteoarthritis susceptibility in ADAMTS-4-knockout mice. Arthritis and rheumatism. 2004,50(8):254758.

43. Glasson SS, Askew R, Sheppard B, Carito B, Blanchet T, Ma HL, et al. Deletion of active ADAMTS5 prevents cartilage degradation in a murine model of osteoarthritis. Nature. 2005,434(7033):644-8.

44. Zhang Q, Ji Q, Wang X, Kang L, Fu Y, Yin Y, et al. SOX9 is a regulator of ADAMTSs-induced cartilage degeneration at the early stage of human osteoarthritis. Osteoarthritis and cartilage. 2015,23(12):2259-68.

\section{Tables}

Table1. Characteristics of experiment $\operatorname{subjects}\left(\chi^{2}\right.$ gender $=2.602, \mathrm{~F}_{\mathrm{age}}=2.940$, both $\left.P \otimes 0.05\right)$ 


\begin{tabular}{|llllllllll|}
\hline OA & & & KBD & & \multicolumn{5}{c|}{ Control } \\
\hline $\begin{array}{l}\text { Sample } \\
\text { set }\end{array}$ & Gender & Age & $\begin{array}{l}\text { Sample } \\
\text { set }\end{array}$ & Gender & Age & $\begin{array}{l}\text { Sample } \\
\text { set }\end{array}$ & Gender & Age \\
\hline 2 & F & 59 & 1 & M & 62 & 1 & F & 45 \\
\hline 3 & F & 62 & 2 & M & 56 & 2 & F & 59 \\
\hline 4 & M & 45 & 3 & M & 51 & 3 & M & 37 \\
\hline 5 & F & 63 & 4 & F & 63 & 4 & M & 46 \\
\hline 6 & F & 52 & 5 & M & 63 & 5 & F & 56 \\
\hline 7 & M & 56 & 6 & F & 59 & 6 & F & 62 \\
\hline 8 & M & 60 & 8 & M & 54 & 7 & M & 39 \\
\hline 9 & F & 65 & 9 & M & 60 & 9 & F & 70 \\
\hline & & & 10 & M & 57 & 10 & F & 42 \\
\hline Mean & - & 57.3 & Mean & - & 57.1 & Mean & - & 50.5 \\
\hline
\end{tabular}

Abbreviation. OA Osteoarthritis $₫ K B D$ Kashin-beck disease, Control Normal people, F Female, M male

Figures 

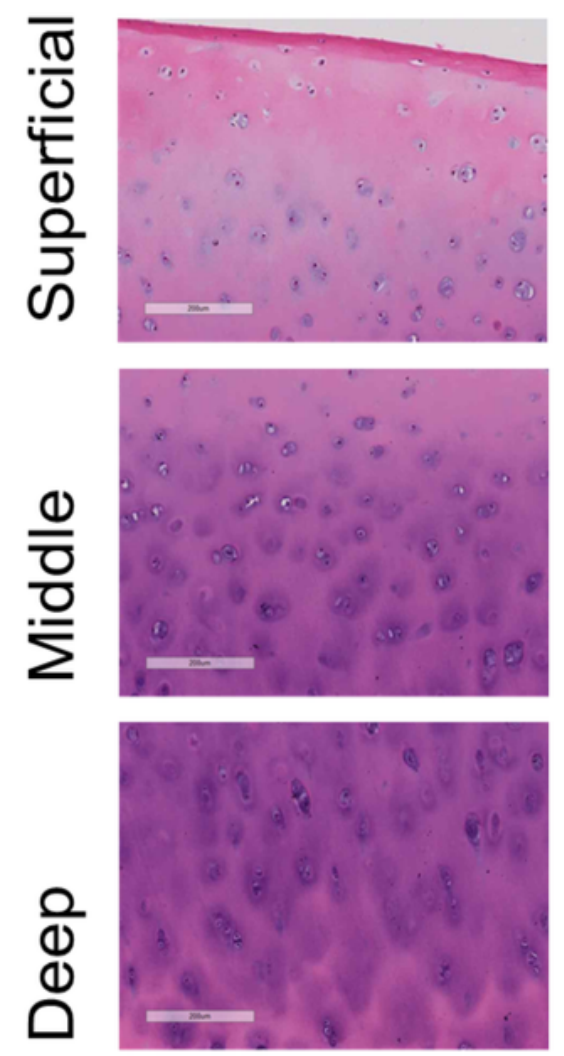

$\mathrm{OA}$
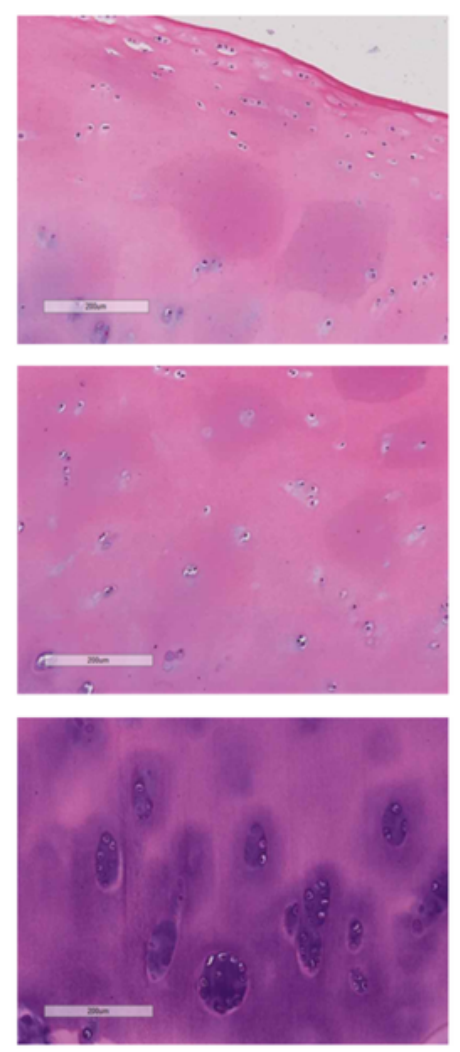

KBD
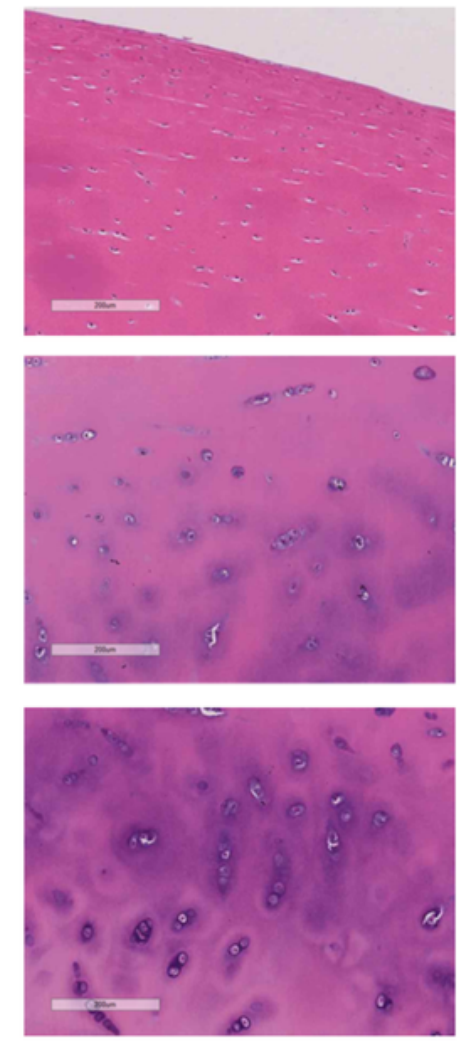

Control

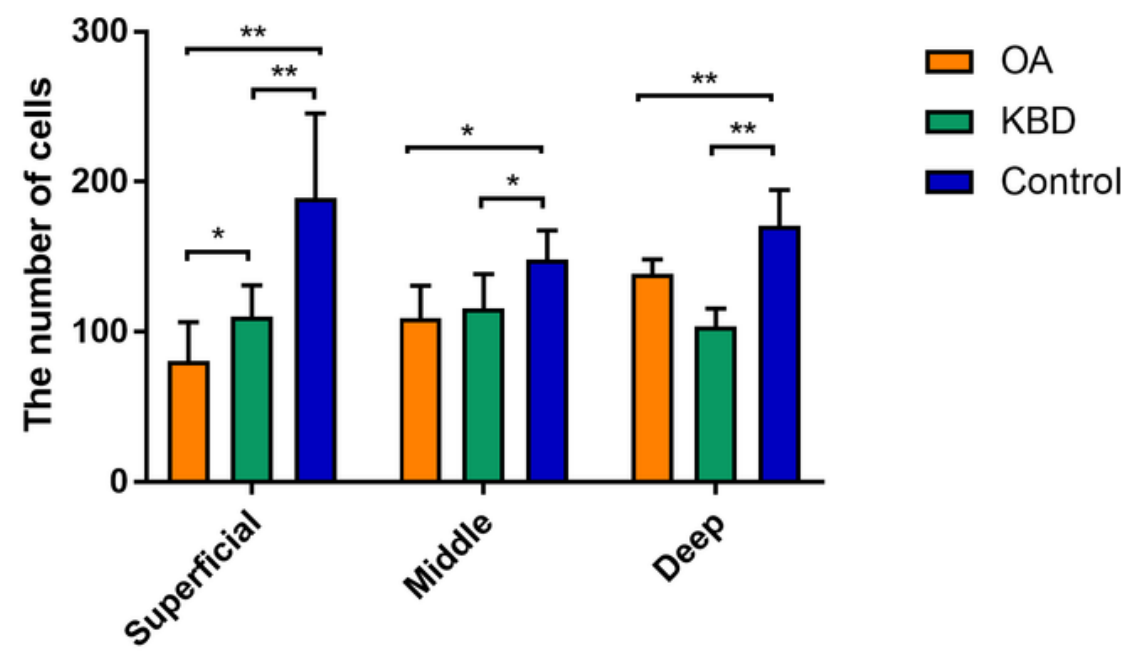

Figure 1

Representative H\&E staining of articular cartilage from an OA patient, a KBD patient, a normal (control). Note. The number of living chondrocytes in $\mathrm{OA}$ and KBD groups were decreased among three zones compared with the control group. ( ${ }^{\star} \mathrm{P}<0.05,{ }^{*} \mathrm{P}<0.01$, scale bar $200 \mu \mathrm{m}$.) 

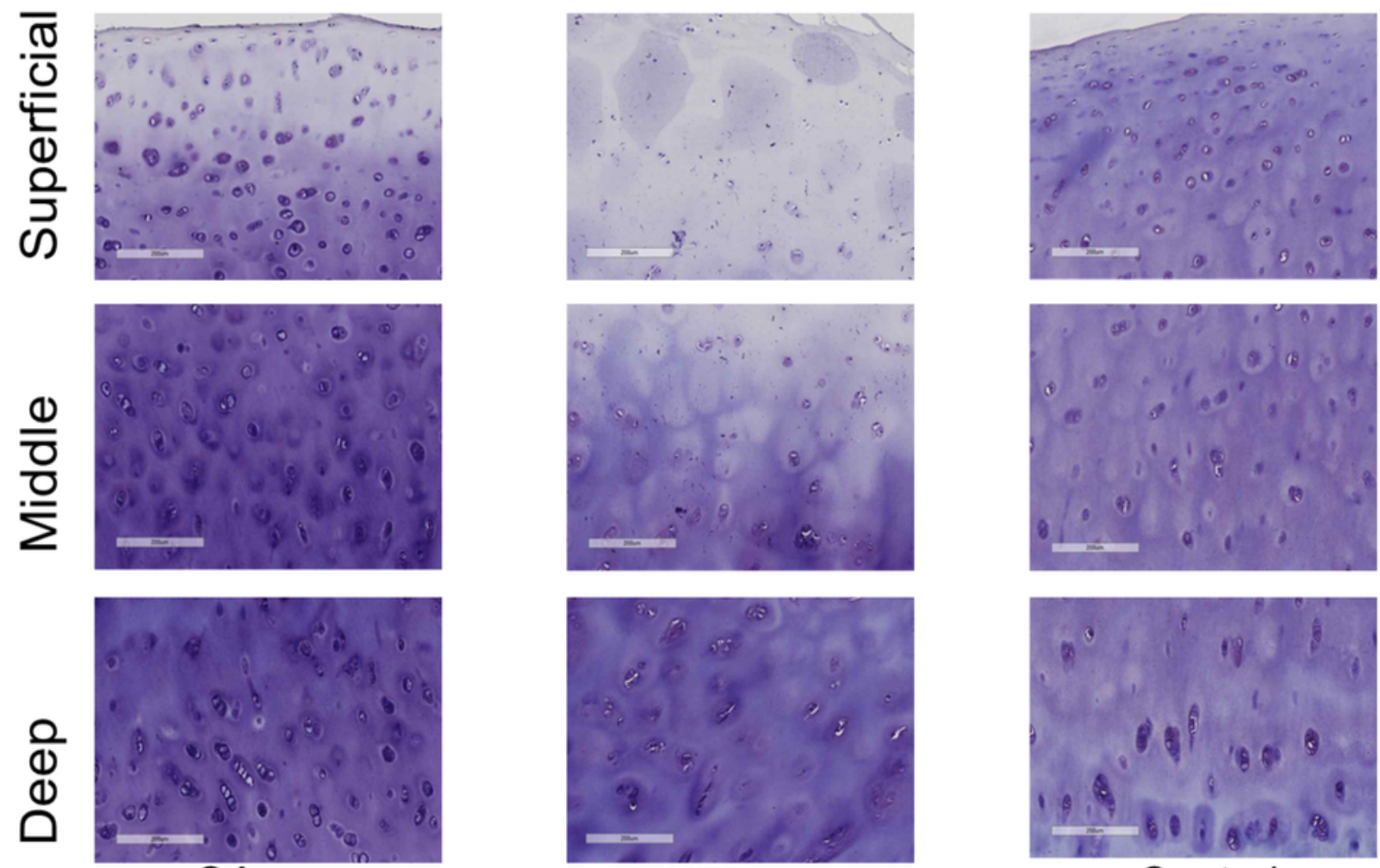

$\mathrm{OA}$

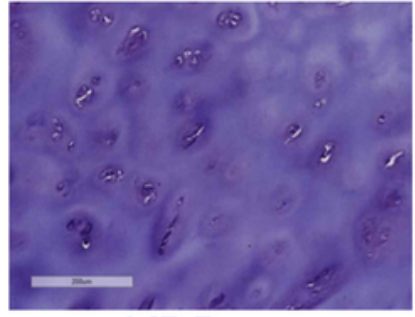

KBD

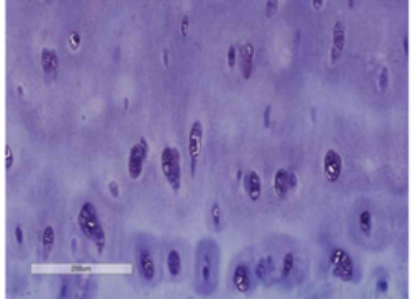

Control

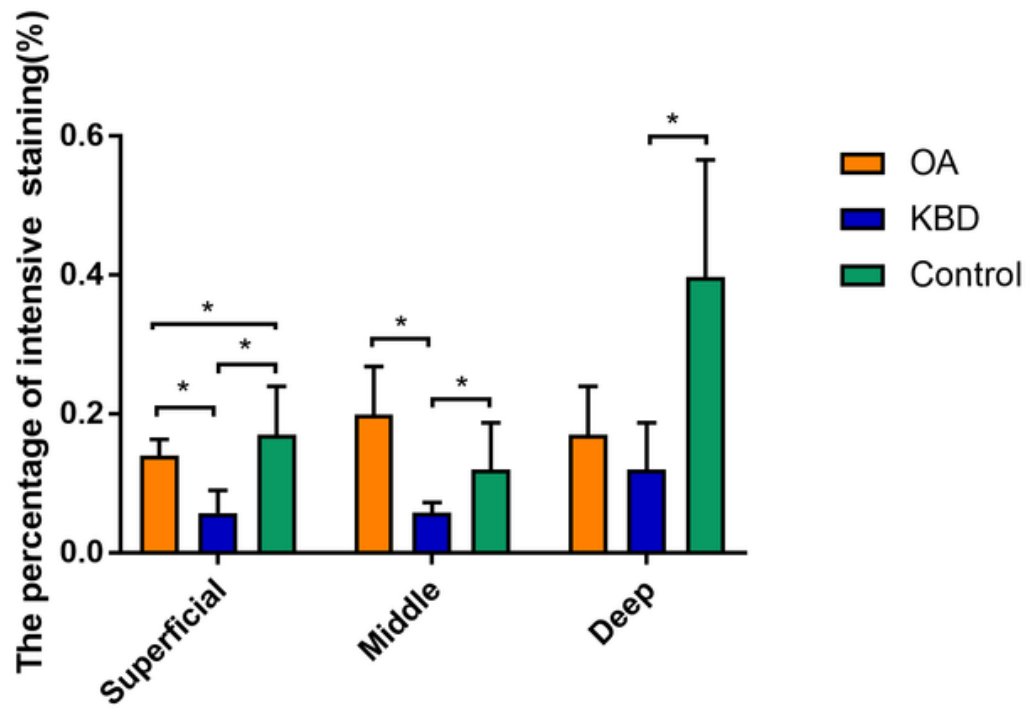

Figure 2

Representative TB staining of articular cartilage from an OA patient, a KBD patient, a normal (control). Note. The average percentage of intensive staining areas in KBD group was significantly decreased compared with the control group. ( ${ }^{\star} \mathrm{P}<0.05,{ }^{\star *} \mathrm{P}<0.01$, scale bar $200 \mu \mathrm{m}$.) 

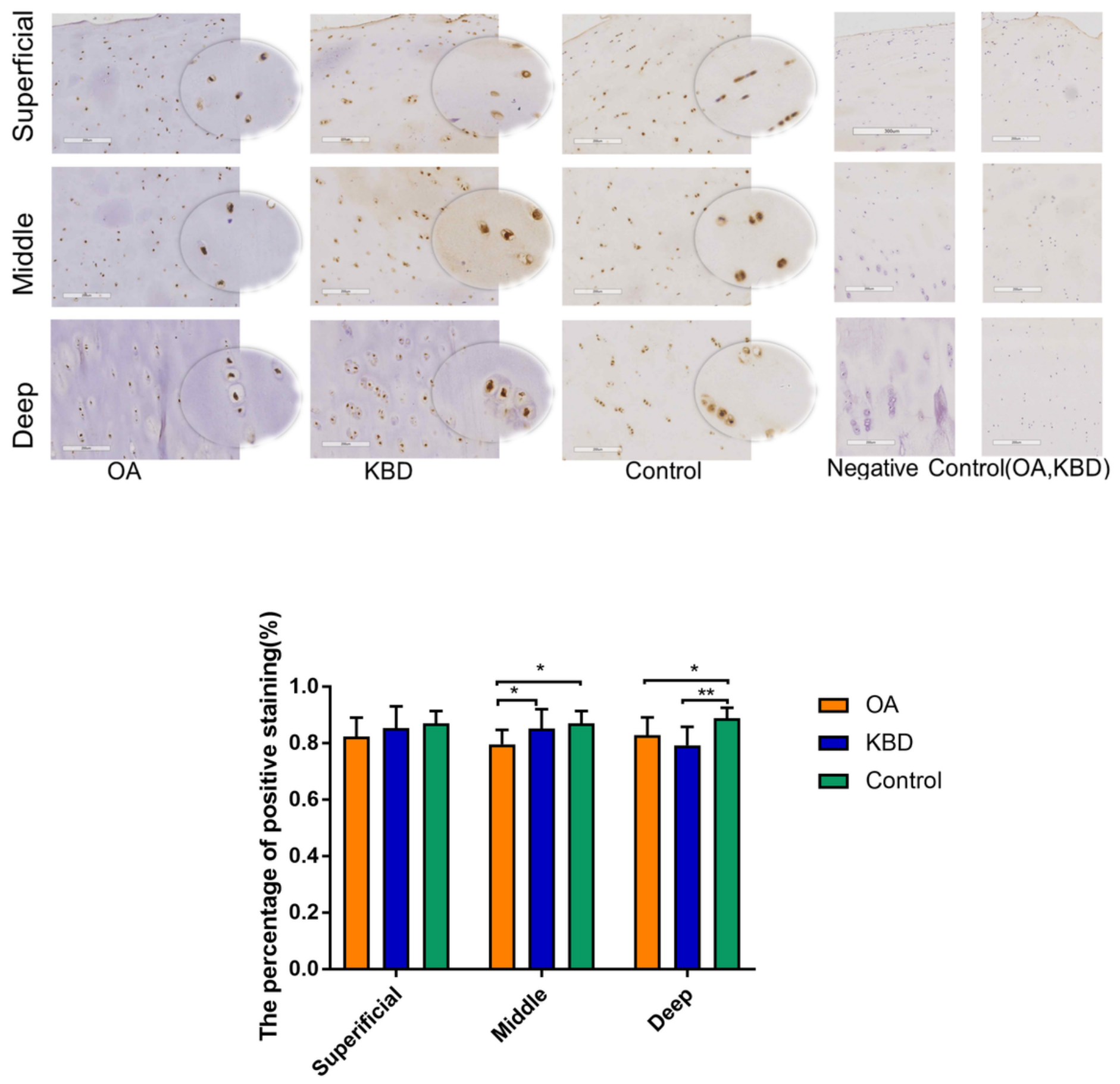

Figure 3

Representative IHC staining of ADAMTS4 (positive staining is in brown) of articular cartilage from OA group, KBD group, and control group among superficial, middle and deep zones. Note. The primary antibody was replaced with PBS in negative control. In superficial zone, there are no obvious difference among three groups. In middle and deep zones, OA and KBD groups increased as compared with control group. ( ${ }^{*}<0.05$, ${ }^{\star *} P<0.01$, scale bar $200 \mu \mathrm{m}$, the round parts showed the staining more clearly with 200×magnification) 

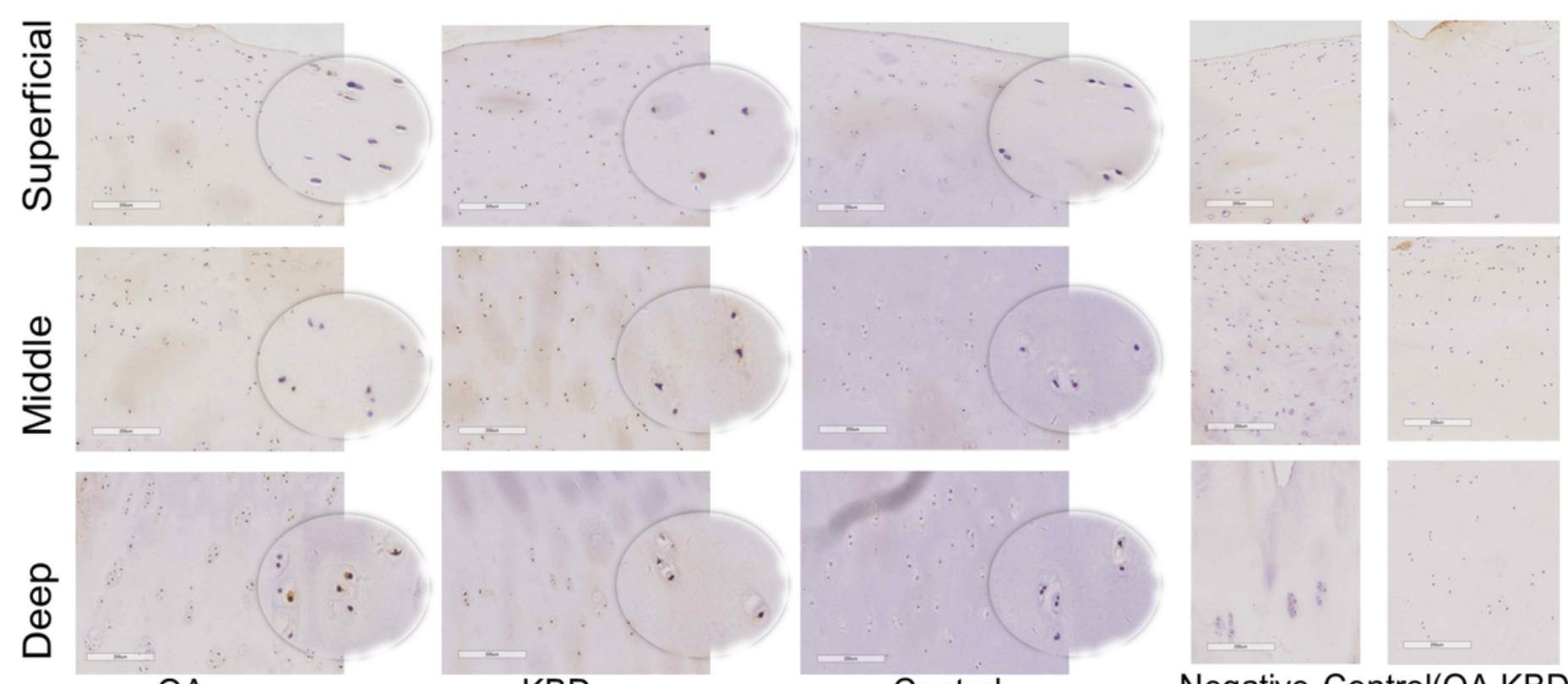

$\mathrm{OA}$

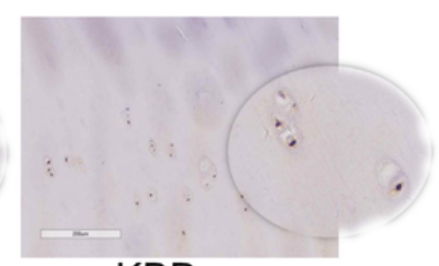

KBD

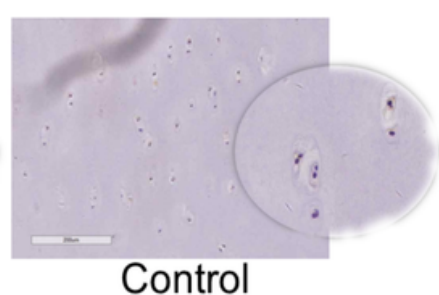

Control

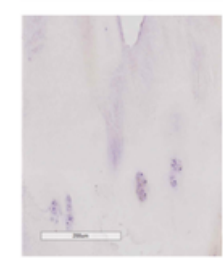

Negative Control(OA,KBD)

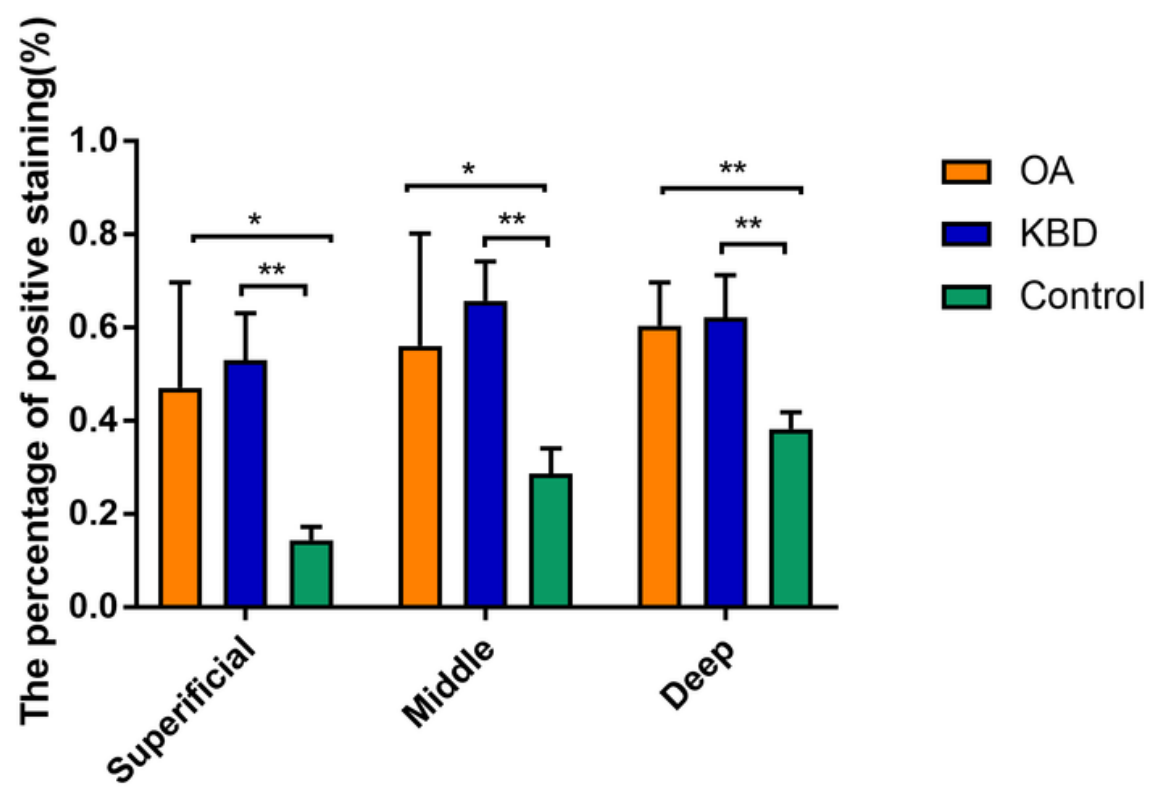

Figure 4

Representative IHC staining of ADAMTS5 (positive staining is in brown) of articular cartilage from OA group, KBD group, and control group among superficial, middle and deep zones. Note. The primary antibody was replaced with PBS in negative control. Among three zones, a significantly increased number of ADAMTS5 positive-stained chondrocytes was observed of OA and KBD cartilage compared to the control. There are also increasing trends in three groups from superficial to deep zones. $\left({ }^{\star} P<0.05, \star \star\right.$ $P<0.01$, scale bar $200 \mu \mathrm{m}$, the round parts showed the staining more clearly with $200 \times$ magnification) 